\title{
REPRESENTATION OF LINEAR SETS AS CRITICAL SETS
}

\author{
ROBERT KAUFMAN
}

ABstract. A class of linear sets investigated by Besicovitch and Taylor is related to the critical set of differentiable mappings of a specified degree of smoothness. An example is constructed to show that certain results on Hausdorff measure are nearly best-possible.

Let $F$ be a compact set of real numbers and $[a, b]$ the smallest interval containing $F$. The complement $[a, b] \sim F$ is composed of a countable sequence of disjoint open intervals, of lengths $l_{n}$. We investigate sets $F$ of Lebesgue measure 0 with the property that $\sum l_{n}^{c}<\infty$ for some $c$ in $(0,1)$. These sets were considered by Besicovitch and Taylor in [1] but our theorems are in a different direction.

We require a class of functions $C^{\beta}$ defined for each number $\beta>1$ : a real function $f$ on an interval is of class $C^{\beta}$ provided it is $n$ times continuously differentiable, where $n<\beta \leqq n+1$, and $D^{n} f$ is of class $\mathrm{Lip}^{\beta-n}$. When $\beta=n+1$ this conflicts with the usual definition of $C^{n+1}$, but no confusion is to be expected; in fact by allowing a larger class $C^{n+1}$ we obtain a slightly sharper result.

Theorem 1. Let $f$ belong to $C^{\beta}$, let $Z$ be the zero-set of $D f$, and let $F=f(Z)$. Then $F$ has Hausdorff $1 / \beta$-measure 0 , and the lengths $l_{n}$ fulfill the condition $\sum l_{n}^{1 / \beta}<\infty$.

Theorem 2. Conversely, let $F$ be a compact set of Lebesgue measure 0 , whose contiguous intervals fulfill the convergence condition above. Then $F=f(Z)$ for some function $f$ in $C^{\beta}$ for which $D f \geqq 0$ and whose zeroset $Z$ has Lebesgue measure 0 . When $\beta=n+1, f$ can be made $n+1$ times continuously differentiable.

Notation. The diameter of a set $E$ is written $|E|$, and its Lebesgue measure $m(E)$. The modulus of continuity of a function $f$ on a set $T$ is defined for $u>0$ as

$$
w(u)=\sup \left|f\left(t_{1}\right)-f\left(t_{2}\right)\right|:\left|t_{1}-t_{2}\right| \leqq u .
$$

Then $w(u) \ll u^{c}$ defines the class Lip ${ }^{c}, 0<c \leqq 1$.

1. The proof of Theorem 1 is largely a variant of Taylor's theorem,

Received by the editors December 1, 1969.

AMS Subject Classifications. Primary 2649, 2880.

Key Words and Phrases. Hausdorff measure, contiguous intervals, modulus of continuity. 
the object being to exploit the extra information on the highest-order derivative.

Lemma 1. Let $f$ be $k$ times continuously differentiable on an interval $[c, d]$ and let $D f, \cdots, D^{k} f$ vanish at least once in the interval. Then

$$
\int_{c}^{d}|D f(t)| d t \leqq(d-c)^{k-1} \int_{0}^{d-c} w(u) d u,
$$

where w is the modulus of continuity of $D^{k} f$.

Proof. For $k=1$ and $D f(\xi)=0, c \leqq \xi \leqq d$, we have

$$
\int_{c}^{d}|D f(t)| d t \leqq \int_{0}^{\xi-c}+\int_{0}^{d-\xi} w(u) d u \leqq \int_{0}^{d-c} w(u) d u .
$$

Assuming the truth of the lemma for $k-1 \geqq 1$,

$$
\int_{c}^{d}\left|D^{2} f(t)\right| d t \leqq(d-c)^{k-2} \int_{0}^{d-c} w(u) d u .
$$

Because $D f$ has a zero in $[c, d],|D f| \leqq \int_{c}^{d}\left|D^{2} f(t)\right| d t$ and the lemma follows from this.

To prove Theorem 1, we observe first that each interval $I=\left(t_{1}, t_{2}\right)$ contiguous to $f(Z)$ has the form $f(J)$ for some interval $J$ contiguous to $Z$. Indeed, let $s_{1} \in f^{-1}\left(t_{1}\right)$ and $s_{2} \in f^{-1}\left(t_{2}\right)$ be so chosen that $\left|s_{1}-s_{2}\right|$ attains its minimum value. Then the interval $J$ between $s_{1}$ and $s_{2}$ is mapped into $I$, and therefore on to $I$. Thus it is sufficient to prove that $\sum|f(J)|^{1 / \beta}<\infty$, where the summation is extended to intervals $J$ contiguous to $Z$.

First, let $Z^{\prime}$ be the derived set of $Z$ and let $J$ have at least one end point in $Z^{\prime}$. Then $D f, \cdots, D^{n} f$ vanish there and by Lemma 1

$$
|f(J)|=\left|\int_{J} D f\right| \leqq|J|^{n-1} \int_{0}^{|J|} w(u) d u \ll|J|^{\beta} .
$$

To treat the isolated points in $Z$, let $J$ be an interval contiguous to $Z^{\prime}$, so that if $J$ meets $Z$ then $J \cap Z$ is discrete. Thus $J \cap Z$ can be enumerated $\cdots<z_{-1}<z_{0}<z_{1}<\cdots$, and we must estimate the sum $\cdots+\left|f\left(z_{1}\right)-f\left(z_{0}\right)\right|^{1 / \beta}+\cdots$. The sequence $z_{-1}<z_{0}<z_{1}<\cdots$ can be arranged into disjoint blocks of exactly $n+1$ terms, with a possible remainder of at most $n$ terms. By Rolle's Theorem we know that $D^{2} f, \cdots, D^{n} f$ each have zeros on any interval $\left[z_{i}, z_{i+n}\right]$, whence $\int_{z_{i}}^{z_{i+n}}|D f| \ll\left|z_{i+n}-z_{i}\right|^{\beta}$. The same estimate can be made for the remainder allowed before, because one of the extreme terms is succeeded immediately by an element of $Z^{\prime}$. Applying the inequality 


$$
\sum_{0}^{n} x_{i}^{1 / \beta} \leqq(n+1)^{1-1 / \beta}\left(\sum_{0}^{n} x_{i}\right)^{1 / \beta}
$$

we find that

$$
\cdots+\left|f\left(z_{1}\right)-f\left(z_{0}\right)\right|^{1 / \beta}+\cdots \ll|J| .
$$

The estimation given applies to all but $2 n$ intervals situated entirely to one side of $Z^{\prime}$, and the proof is complete. (The possibility that $Z^{\prime}=\varnothing$ makes the last remark necessary.)

That $F$ has Hausdorff $1 / \beta$-measure 0 is proved very simply in [1], but we present a less elementary proof for a stronger conclusion.

LeMma 2. Let $f$ be absolutely continuous on an interval $[c, d]$ and let $\int_{A}|D f|=0$ for a closed subset $A \subseteq[c, d]$. Suppose that for every interval $J$ contiguous to $A$,

$$
\int_{J}|D f| \ll|J|^{\beta} \text { for a certain real number } \beta>1 .
$$

Then $f(A)$ is contained in $o(N)$ intervals of length $N^{-\beta}, N \rightarrow+\infty$.

Proof. We shall replace $f$ by a function $g$ that coincides with $f$ on $A$, and is again absolutely continuous. To do so we define $D g$ on the intervals $J$ so that $\int_{J} D g=\int_{J} D f$. Thus, when $J=\left(t_{1}, t_{2}\right)$, set

$$
\begin{aligned}
& D g(s)=c\left(s-t_{1}\right)^{\beta-1}, \quad t_{1}<s \leqq \frac{1}{2}\left(t_{1}+t_{2}\right), \\
& D g(s)=c\left(t_{2}-s\right)^{\beta-1}, \quad \frac{1}{2}\left(t_{1}+t_{2}\right)<s<t_{2}
\end{aligned}
$$

for a constant $c$. Then in fact

$$
c=2^{\beta-1}\left(t_{2}-t_{1}\right)^{-\beta}\left(f\left(t_{2}\right)-f\left(t_{1}\right)\right) \ll 1,
$$

whence

$$
D g(s): \ll(\operatorname{dist}(s, A))^{\beta-1} .
$$

Let $I_{N}$ denote any of the intervals $\left[k N^{-1},(k+1) N^{-1}\right]$ that meet $A$, so that $f(A)=g(A) \subseteq \mathrm{U} g\left(I_{N}\right)$. On each $I_{N}$ we have $|D g| \ll N^{1-\beta}$, so that $\left|f\left(I_{N}\right)\right| \ll N^{-\beta}$. Of course, the number of intervals $I_{N}$ is $O(N)$.

Fixing a number $\epsilon<1$ we divide the intervals $I_{N}$ into two classes.

(i) $m\left(I_{N} \cap A\right)>(1-\epsilon) N^{-1}$. In this event every point of $I_{N}$ is within $\epsilon N^{-1}$ of $A$, and this allows us to introduce a factor $\epsilon^{\beta-1}$ into the previous estimate of $\left|g\left(I_{N}\right)\right|$, still preserving the number of intervals $I_{N}$.

(ii) $m\left(I_{N} \cap A\right) \leqq(1-\epsilon) N^{-1}$. Let us write $\nu_{N}$ for the number of the intervals, and $\nu_{N}^{\prime}$ for the number treated in (i). Then $m(A) \leqq N^{-1} \nu_{N}^{\prime}$ $+(1-\epsilon) N^{-1} \nu_{N}$. But because $A$ is closed, $\nu_{N}+\nu_{N}^{\prime}=N m(A)+o(N)$, 
hence $\nu_{N}=o(N)$. Lemma 2 is an easy consequence of this fact and the estimate given in (i).

To obtain the result on the $1 / \beta$-measure of $f(Z)$ we select $A=Z^{\prime}$ and note that $Z \sim Z^{\prime}$ is countable. It is worth remarking that if $D f \geqq 0$ and $m(Z)=0$ then $f$ is strictly monotone and $f\left(Z \sim Z^{\prime}\right)$ is the set of isolated points of $f(Z)$.

2. In this section we suppose that $F$ is a set described in Theorem 2. Let $g$ be absolutely continuous on $[a, b] \supseteq F$, and linear on each contiguous interval $J$, with derivative $|J|^{1 / \beta-1}$. The mapping inverse to $g$, say $h$, is increasing and continuous because $D g>0$ almost everywhere. But $h$ is also absolutely continuous because it maps each (Lebesgue) null set onto a null set. Thus $F$ is subject to the previous lemma, since $|g(J)|=|J|^{1 / \beta}$ and $m(g(F))=0$.

In the proof of Theorem 2 we keep the function $h$, but regard it solely as a mapping of $g(F)$ onto $F$. We now extend $h$ to a mapping of class $C^{\beta}$. Let $\chi$ be a function in $C^{\infty}[0,1]$,

$\chi(0)=0, \quad \chi(1)=1, \quad D \chi>0$ on $(0,1), \quad D^{k} \chi(0)=D^{k} \chi(1)=0$,

$$
1 \leqq k<\infty \text {. }
$$

On each interval $\left(t_{1}, t_{2}\right)$ contiguous to $g(F)$ we define

$$
f(s)=h\left(t_{1}\right)+\left(t_{2}-t_{1}\right)^{\beta} \chi\left(\left|s-t_{1}\right| /\left(t_{2}-t_{1}\right)\right), \quad t_{1}<s<t_{2} .
$$

Then $f\left(t_{1}+\right)=h\left(t_{1}\right), f\left(t_{2}-\right)=h\left(t_{1}\right)+\left(t_{2}-t_{1}\right)^{\beta}=h\left(t_{2}\right)$. When $1 \leqq k<\infty$,

$$
D^{k} f(s)=\left(t_{2}-t_{1}\right)^{\beta-k} D^{k} \chi\left(\left|s-t_{1}\right| /\left(t_{2}-t_{1}\right)\right) .
$$

In particular the $k$ th derivative of $f$, on the complement of $g(F)$, is uniformly bounded for $1 \leqq k \leqq \beta$.

Now $f$ is absolutely continuous, for it is monotone-increasing and continuous, and preserves null sets. Hence its derivative is given by $D f$ (extended to all of $h([a, b])$. Also, the functions $D f, \cdots, D^{n-1} f$ are continuous on $h[a, b]$, vanish on $h(F)$, and have uniformly bounded derivatives on the complement of $h(F)$. It follows that each is the derivative of its predecessor; for the same reasons $D^{n} f$ is the derivative of $D^{n-1} f$, and $f$ is $n$ times continuously differentiable. From the formula for $D^{n} f$, it vanishes continuously on $h(F)$, and when $\beta<n+1, D^{n} f$ satisfies a Lipschitz condition of order $\beta-n$, on the contiguous intervals. From these facts the Lipschitz condition for all of $h([a, b])$ is easily deduced.

To improve this result for $\beta=n+1$, we proceed as follows. Writing $l_{1} \geqq l_{2} \geqq \cdots \geqq l_{n} \geqq \cdots$ for the lengths of intervals $I_{n}$ complementary to $F$, we find numbers $1<c_{1}<c_{2}<\cdots<c_{n} \rightarrow+\infty$ such that 
$\sum\left(c_{n} l_{n}\right)^{1 / \beta}<\infty$. We then modify the function $g$, so that $I_{n}$ is mapped onto an interval of length $\left(c_{n} l_{n}\right)^{1 / \beta}$. The function $h$ inverse to $g$ is also modified and so ultimately is the function $f$ (constructed with the aid of the auxiliary mapping $\chi$ ). We consider in detail this function, $\tilde{f}$.

Writing $\left(t_{1}, t_{2}\right)$ for the transform by $g$ of the interval $I_{n}$, we have

$$
\begin{aligned}
t_{2} & =t_{1}+\left(c_{n} l_{n}\right)^{1 / \beta}, \\
\tilde{f}(s) & =h\left(t_{1}\right)+\bar{c}_{n}^{-1}\left(t_{2}-t_{1}\right)^{\beta} \chi\left(\left|s-t_{2}\right| /\left(t_{2}-t_{1}\right)\right), \quad t_{1}<s<t_{2}, \\
D^{n+1} \tilde{f}(s) & =c_{n}^{-1} D^{n+1} \chi\left(\left|s-t_{2}\right| /\left(t_{2}-t_{1}\right)\right) .
\end{aligned}
$$

Since the factor $c^{-1}$ converges to 0 with the length of the interval $\left(t_{1}, t_{2}\right), \hat{f}$ belongs to the conventional class $C^{n+1}$.

3 . In this section we show that the vanishing of the $1 / \beta$-measure of $f(Z)$ cannot be strengthened very much. Let $q$ be a function on $(0$, $\infty)$ such that $q(t)$ and $t^{1 / \beta} / q(t)$ are increasing, $\sum_{1}^{\infty} q\left(2^{-m}\right)<\infty$.

THEOREM 3. There exists a function $f$ satisfying the conditions of Theorem 1, for which $f=F(Z)$ has positive Hausdorff measure with respect to the function $\phi(t)=t^{1 / \beta} / q(t)$.

Choosing $q(t)=\log ^{2}\left(t^{-1}\right)$ for small $t$, we find that $F(Z)$ can have dimension $1 / \beta$.

Proof. Without loss of generality we can suppose $\sum_{1}^{\infty} q\left(2^{-m}\right)<1$. In each dyadic interval $\left[k 2^{-m},(k+1) 2^{-m}\right] \subseteq[0,1]$ we construct an interval centered at $\left(k+\frac{1}{2}\right) 2^{-m}$, of length $2^{-m} q\left(2^{-m}\right)$. We remove all intervals defined for $m=1$, then all intervals defined for $m=2$ save those intersecting an interval already removed, and so on. The disjoint intervals selected form an open set $W$ of measure $m(W)<1$. Let $D f=0$ on $Z=[0,1] \sim W$, and on an interval $I$ of $W$, let $D f$ $=|I|^{\beta-1}$. Then $f(Z)$ is a set $F$, since the contiguous intervals have lengths $|I|^{\beta}$ corresponding to the components $I$ of $W$.

Observe next that if $s_{1}<s_{2}$ and $s_{1}, s_{2} \in Z$, the $f\left(s_{2}\right)-f\left(s_{1}\right)$ $\gg\left(s_{2}-s_{1}\right)^{\beta} q^{\beta}\left(s_{2}-s_{1}\right)$. Indeed $\left(s_{1}, s_{2}\right)$ contains a dyadic interval $\left[k 2^{-m},(k+1) 2^{-m}\right]$, with $2^{-m} \geqq \frac{1}{4}\left(s_{2}-s_{1}\right)$. The interval constructed in $\left[k 2^{-m},(k+1) 2^{-m}\right]$ either belongs to $W$, or intersects a larger interval contained in $W$, of length $\geqq 2^{-m} q\left(2^{-m}\right) \gg\left(s_{2}-s_{1}\right) q\left(s_{2}-s_{1}\right)$. In any case an interval of that length belongs entirely to $W \cap\left(s_{1}, s_{2}\right)$, whence the lower bound on $f\left(s_{2}\right)-f\left(s_{1}\right)$.

Let $\mu$ be the measure of Borel sets $E$ defined by

$$
\mu(E)=m\left(Z \cap f^{-1}(E)\right), \quad \mu(f(Z))=m(Z)>0 .
$$

The proof will be completed by showing that $\mu(I) \ll \phi(|I|)$ for all 
intervals $I$. Now $I$ contains a subinterval $I_{0}$ with end points in $f(Z)$, such that $\mu\left(I_{0}\right)=\mu(I)$, and of course $\phi\left(\left|I_{0}\right|\right) \leqq \phi(|I|)$.

Let $I_{0}=f(J)$, for an interval $J$ contiguous to $W$. Then $\mu\left(I_{0}\right) \leqq|J|$, while $\left|I_{0}\right| \gg \mid J{ }^{\beta} q^{\beta}(|J|)$. Thus

$$
\mu\left(I_{0}\right) \ll\left|I_{0}\right|^{1 / \beta} / q(|J|) \ll\left|I_{0}\right|^{1 / \beta} / q\left(\left|I_{0}\right|\right),
$$

because $\left|I_{0}\right| \ll|J|$.

Related questions in Euclidean space have been treated by Sard in [2].

\section{REFERENCES}

1. A. S. Besicovitch and S. J. Taylor, On the complementary intervals of a linear closed set of zero Lebesgue measure, J. London Math. Soc. 29 (1954), 449-459. MR 16, 344.

2. A. Sard, Images of critical sets, Ann. of Math. (2) 68 (1958), 247-259. MR 20 \#6499.

UNIVERSITY OF ILLINOIS, URB ANA, ILLINOIS 61801 